\title{
Non-vacuum perfect fluid static cylindrically symmetric solutions in $f(R)$ gravity and their energy distribution
}

\begin{abstract}
Much attention has been given towards modified theories of gravity especially towards $f(R)$ gravity during the last two decades to understand the reason behind the accelerated expansion of the universe. Recently, Sharif and Sadia explored the non-vacuum static cylindrically symmetric solutions for dust case and their energy contents too. In this paper, we are intend to extend their work for perfect fluid case. Regarding this, we obtain the nonvacuum field equations of $f(R)$ gravity for perfect fluid static solution using cylindrically sym-metric background. The field equations turn out to be complected and hence can't be solved analytically so we have to put some restrictions to solve the field equations. For this purpose, we utilize metric approach and constant curvature assumption. Further, we explore the energy distributions of the obtained solutions by using generalized Landau-Lifshitz energy-momentum prescription in the context of $f(R)$ theory of gravity for a specific choice of $f(R)$ models. Moreover, we examined the stability conditions for the obtained solutions.
\end{abstract}

Keywords: $f(R)$ gravity, vacuum solutions, perfect fluid, static cylindrically symmetric, generalized landau-lifshits complex
Volume 2 Issue 5 - 2018

\author{
Farhat Imtiaz, M Jamil Amir \\ Department of Mathematics, University of Lahore, Sargodha \\ Campus-40100, Pakistan
}

Correspondence: $\mathrm{M}$ Jamil Amir, Department of Mathematics, University of Lahore, Sargodha Campus-40100, Pakistan, Emailmjamal.dgk@gmail.com

Received: August 28, 2018 | Published: October 25, 2018

\section{Introduction}

Out of many other alternative theories of Einstein's theory of gravity, the $f(R)$ theory of gravity has a long history. During the last few years, there has been tremendous change in this area bringing about a lot of study which yields exciting results. On behalf of some evidences, it has been concluded that Weyl ${ }^{1}$ was the first who worked in the field of $f(R)$ gravity while many others are in view that it was Eddington ${ }^{2}$ who was the pioneer to contribute in this field. Later, Buchdahl ${ }^{3}$ reflected his ideas and efforts in the same field. A comprehensive review has been presented here which consists of relevant literature and important features and characteristics of $f(R)$ theories of gravity. ${ }^{4}$

Using the relation with the week field limit, Capozziello et al. ${ }^{5}$ obtained exact spherical symmetric solutions in $f(R)$ gravity for constant Ricci scalar as well as for Ricci scalar as function of radial coordinates. Later on, the same authors ${ }^{6}$ discussed spherically symmetric solution in $f(R)$ gravity via Noether symmetric approach. Kainulanianen et al. ${ }^{7}$ investigated spherically symmetric spacetimes in $f(R)$ theories of gravity using analytical and numerical approaches. Multamaki and Vilja ${ }^{8}$ explored exact spherically symmetric static empty space solution. These results showed that a huge number of $f(R)$ theories have exact solutions as Schwarzschild de sitter metric. The said authors ${ }^{9}$ also extracted non-vacuum solutions using static spherical symmetric background.

Hollenstein and Lobo ${ }^{10}$ coupled $f(R)$ gravity to non-linear electrodynamics in order to produce static spherically symmetric solutions. In an extended study of $f(R)$ gravity, Shojai ${ }^{11}$ pondered on static spherically symmetric interior solutions. Caramês and Mello ${ }^{12}$ in a higher dimensional spacetime, scrutinizes spherically symmetric vacuum solutions. Sharif and Kousar ${ }^{13}$ determined non-vacuum static spherically symmetric solutions. Much work is available in literature carried out by different authors. ${ }^{14-18}$
In a detailed study of $f(R)$ gravity, Sharif and Shamir ${ }^{19}$ researched static plane symmetric vacuum solutions. Amir and Maqsood $^{20}$ discussed some non-vacuum plane symmetric solutions using metric approach and non-varying Ricci scalar assumption. They also explored the energy contents of these solutions. Shamir ${ }^{21}$ has an elaborated contributions in examining plane symmetric vacuum Bianchi type III cosmology in $f(R)$ gravity. Momeni and Gholizade ${ }^{22}$ proved that exact solution of $f(R)$ gravity constant curvature can be applied to the exterior of a string. Azadi et al. ${ }^{23}$ using Weyl coordinates in the framework of the metric $f(R)$ theories of gravity, explored the static cylindrically symmetric vacuum solutions.

Amir and Sattar ${ }^{24}$ found locally rotationally symmetric vacuum solutions in $f(R)$ models. Amir and Naheed ${ }^{25}$ explored vacuum solutions of spatially homogeneous rotating spacetimes. Sharif and Arif ${ }^{26}$ worked on dust particle for the investigation of static cylindrically symmetric solutions in metric $f(R)$ gravity.

In GR , energy localization is a very serious problem and it is not still sorted out exactly. Much work has been done on problem in the framework of GR to resolve this issue. Different scientists gave their own energy momentum complexes and found the energy momentum distribution of many spactimes but could not present a concrete conjuncture. Methodology of energy-momentum pseudo tensor was the first effort to solve this matter and this step was taken by Einstein. Following him many authors, like Landau-Lifshitz, Moller Bergmann-Thomson and Weinberg gave their own energymomentum prescriptions. Virbhadra and Parikh $^{27}$ investigated the energy-momentum distribution of several spacetimes, such as KerrNewmann, Kerr-Schild classes, Einstein-Rosen, Vaidya and BonnorVaidya spacetimes but could not reach at solid and unique conclusion.

There are many authors who are of the view point that this issue may be tackled correctly in the other frameworks, for example, in 
telepararllel(TP) theory of gravity, modified theories of gravity etc. In the framework of TP gravity, Sharif and Amir ${ }^{28-34}$ found out energymomentum distribution of various spacetimes by using TP version of different prescriptions. They conclude that no general conjecture can be made here because results in some cases are consistent while others are inconsistent. Landau-Lifshitz energy momentum complex was generalized to evaluate energy-momentum distribution for Schwarzschild de Sitter spacetime. The energy density was calculated with the help of generalized Landau-Lifshitz prescription for the plane symmetric static solution and cosmic string space time by Sharif and Shamir ${ }^{35}$.

This paper of current study explores the non-vacuum perfect fluid static cylindrically symmetric solutions by using some basics of $f(R)$ gravity. We utilize generalized Landau-Lifshitz EMC to evaluate energy density for the solution having constant scalar curvature. The following arrangement has been used in this paper. In section 2, the modification of field equations in metric approach of $f(R)$ gravity has been precisely discussed. Section 3 contains the derivation of generalized Landau-Lifshitz EMC. In section 4, the exact non vacuum solution by using the constant curvature condition, of perfect fluid static cylindrically symmetric solutions is described. In section 5 , by using Landau-Lifshitz EMC, we have calculated the energy density of the solution that was obtain in the previous section. In the last section results have been summarized.

\section{Field equations in $f(R)$ theory of gravity}

We will extract the field equations for this section. For this reason, we utilize the metric approach of $f(R)$ theory of gravity. In this approach, the variation of the action is done with respect to the metric tensor only. The action of $f(R)$ theory is expressed as

$$
S=\int \sqrt{-g}\left(\frac{1}{16 \pi G} f(R)+L_{m}\right) d^{4} x,
$$

where $f(R)$ is a arbitrary function of Ricci scala $R$ and $L_{m}$ is the matter Lagrangian. In the standard Einstien-Hilbert action the replacement of $R$ by $f(R)$ gives us this action. By varying this action with respect to metric tensor $g_{\mu v}$, these corresponding field equations can be derived,

$$
F(R) R_{\mu \nu}-\frac{1}{2} f(R) g_{\mu \nu}-\nabla_{\mu} \nabla_{v} F(R)+g_{\mu v} F(R)=\kappa T_{\mu v},
$$

Where

$$
F(R)=\frac{d f(R)}{d R}, f=\nabla^{\mu} \nabla_{\mu} .
$$

Here, $\nabla_{\mu}$ represents the covariant derivative, $f=\nabla^{\mu} \nabla_{\mu}$ is called D' Alembert operator and $T_{\mu \nu}$ is the standard matter energymomentum tensor derived from $L_{m}^{\mu \nu}$. In the metric tensor these are the fourth order partial differential equations. For $f(R)=R$ these equations reduce to the famous Einstein field equations of GR . After contraction the field equations we get are

$$
F(R) R-2 f(R)+3 F(R)=\kappa T
$$

and, in vacuum, i.e., when $T=0$, the last equation turns out be

$$
F(R) R-2 f(R)+3 F(R)=0 .
$$

We come to know an important relationship between $f(R)$ and $F(R)$ from this which helps in the simplification of field equations and to find out the $f(R)$. Any metric with scalar curvature though, as $R=R_{0}$, is a solution of Equation (5) if the below equation is as following

$$
F\left(R_{0}\right) R_{0}-2 f\left(R_{0}\right)=0 .
$$

This condition of the constant scalar curvature for the vacuum and non-vacuum case will have following form

$$
F\left(R_{0}\right) R_{0}-2 f\left(R_{0}\right)=\kappa T .
$$

These conditions have vital role to find the acceptability of $f(R)$ models.

\section{Generalized landau-lifshitz energy- momentum complex}

The generalized Landau-Lifshits EMC is given by

$\tau^{\mu \nu}=f^{\prime}\left(R_{0}\right) \tau_{L L}^{\mu \nu}+\frac{1}{6 \kappa}\left\{f^{\prime}\left(R_{0}\right) R_{0}-f\left(R_{0}\right)\right\} \frac{\partial}{\partial x^{\lambda}}\left(g^{\mu \nu} x^{\lambda}-g^{\mu \lambda}{ }^{v}\right)$

where $\tau_{L L}^{\mu v}$ is the Landau-Lifshitz EMC in GR and $\kappa=8 \pi G$. In the

field of $f(R)$ theory of any metric tensor which holds constant scalar curvature late, EMC can be calculated. Energy density is represented by $00-$ component and as following,

$$
\tau^{00}=f^{\prime}\left(R_{0}\right) \tau_{L L}^{00}+\frac{1}{6 \kappa}\left(f^{\prime}\left(R_{0}\right) R_{0}-f\left(R_{0}\right)\right)\left({\frac{\partial}{\partial x^{i}}}^{00}{ }^{x^{i}}+3 g^{00}\right),
$$

where $\tau_{L L}^{00}$ represents the sum of energy-momentum tensor and the energy-momentum pseudo tensor and is given by

$$
\tau_{L L}^{00}=(-g)\left(T^{00}+t_{L L}^{00}\right)
$$

and

$$
T^{00}=\frac{1}{\kappa}\left(R^{00}-\frac{1}{2} g^{00} R\right),
$$

where $R$ is the Ricci scalar and $t_{L L}^{00}$ can be evaluated from the following expression

$$
\begin{aligned}
& t_{L L}^{00}=\frac{1}{2 \kappa}\left[\left(2 \Gamma_{\alpha \beta}^{\gamma} \Gamma_{\gamma \delta}^{\delta}-\Gamma_{\alpha \delta}^{\gamma} \Gamma_{\beta \gamma}^{\delta}-\Gamma_{\alpha \gamma}^{\gamma} \Gamma_{\beta \delta}^{\delta}\right)\left(g^{\mu \alpha} g^{\nu \beta}-g^{\mu \nu} g^{\alpha \beta}\right)\right. \\
& +g^{\mu \alpha} g^{\beta \gamma}\left(\Gamma_{\alpha \delta}^{v} \Gamma_{\beta \gamma}^{\delta}+\Gamma_{\beta \gamma}^{v} \Gamma_{\alpha \delta}^{\delta}-\Gamma_{\gamma \delta}^{v} \Gamma_{\alpha \beta}^{\delta}-\Gamma_{\alpha \beta}^{v} \Gamma_{\gamma \delta}^{\delta}\right) \\
& \left.+g^{v \alpha} g^{\beta \gamma}\left(\Gamma_{\alpha \delta}^{\mu} \Gamma_{\beta \gamma}^{\delta}+\Gamma_{\beta \gamma}^{\mu} \Gamma_{\alpha \delta}^{\delta}-\Gamma_{\gamma \delta}^{\mu} \Gamma_{\alpha \beta}^{\delta}-\Gamma_{\alpha \beta}^{\mu} \Gamma_{\gamma \delta}^{\delta}\right)+g^{\alpha \beta} g^{\gamma \delta}\left(\Gamma_{\alpha \gamma}^{\mu} \Gamma_{\beta \delta}^{v}-\Gamma_{\alpha \beta}^{\mu} \Gamma_{\gamma \delta}^{v}\right)\right] .
\end{aligned}
$$

\section{Perfect fluid static cylindrically symmetric solutions}

In this section, we find the non-vacuum field equations of $f(R)$ theory for the metric representing static cylindrical symmetric spacetimes by using the condition of constant scalar curvature and metric pattern, i.e., $(R=$ constant $)$. The line element representing static cylindrically symmetric spacetimes is given below

$$
d s^{2}=A(r) d t^{2}-B(r) d \rho^{2}-C(r) d \phi^{2}-B d z^{2},
$$


here $A(r), B(r)$ and $C(r)$ are taken as arbitrary functions of $r$ For this line element Ricci scalar turn out to be

$$
R=\frac{A^{\prime \prime}}{A B}-\frac{A^{\prime 2}}{2 A^{2} B}+\frac{B^{\prime \prime}}{B^{2}}-\frac{B^{\prime 2}}{B^{3}}+\frac{C^{\prime \prime}}{B C}-\frac{C^{\prime 2}}{2 B C^{2}}+\frac{A^{\prime} C^{\prime}}{2 A B C} .
$$

where prime shows the derivative with respect to $r$. For perfect fluid the energy-momentum tensor is given as

$$
T_{\mu v}=(\rho+p) u_{\mu} u_{v}-p g_{\mu v},
$$

where $\rho$ is the density of energy and $p$ is the pressure of the fluid and in comoving coordinates the fourth-velocity is given by $u_{\mu}=\sqrt{g_{00}}(1,0,0,0)$. The equation of state given below is satisfied by pressure $p$ and energy density $\rho$.

$p=\omega \rho, 0 \leq \omega \leq 1$

Also, from field Equation (4), we get

$$
f(R)=\frac{3 F(R)+F(R) R-\kappa T}{2} .
$$

Using this value of $f(R)$ in non-vacuum field Equation (2), we have

$$
\frac{F(R) R_{\alpha \alpha}-\nabla_{\alpha} \nabla_{\alpha} F(R)-\kappa T_{\alpha \alpha}}{g_{\alpha \alpha}}=\frac{F(R)-\square F(R)-\kappa T}{4}
$$

In the above equation, the terms on the right hand side are independent of index $\alpha$, so we can write the field equation in the following manner,

$$
A_{\alpha \alpha}=\frac{F(R) R_{\alpha \alpha}-\nabla_{\alpha} \nabla_{\alpha} F(R)-\kappa T_{\alpha \alpha}}{g_{\alpha \alpha}} .
$$

Here $A_{\alpha \alpha}$ is used to represent the traced quantity. By subtracting (00) and (11) components, we get

$$
\frac{A^{\prime} C^{\prime} F}{4 A B C}-\frac{B^{\prime \prime} F}{2 B^{2}}+\frac{B^{\prime 2} F}{2 B^{3}}-\frac{C^{\prime \prime} F}{2 B C}+\frac{C^{\prime 2} F}{4 B C^{2}}+\frac{A^{\prime} B^{\prime} F}{4 A B^{2}}+\frac{B^{\prime} C^{\prime} F}{4 B^{2} C}+\frac{A^{\prime} F^{\prime}}{2 A B}+\frac{B^{\prime} F^{\prime}}{2 B^{2}}-\frac{F^{\prime \prime}}{B}-\kappa(p+\rho)=0 .
$$

Similarly, we get just two independent equations by subtracting (22) and (33) components from (00).

$$
\frac{A^{\prime \prime} F}{2 A B}-\frac{A^{\prime 2} F}{4 A^{2} B}-\frac{C^{\prime \prime} F}{2 B C}+\frac{C^{\prime 2} F}{4 B C^{2}}+\frac{A^{\prime} F^{\prime}}{2 A B}-\frac{C^{\prime} F^{\prime}}{2 B C}-\kappa(p+\rho)=0
$$$$
\frac{A^{\prime \prime} F}{2 A B}-\frac{A^{\prime 2} F}{4 A^{2} B}-\frac{B^{\prime \prime} F}{2 B^{2}}+\frac{B^{\prime 2} F}{2 B^{3}}-\frac{A^{\prime} B^{\prime} F}{4 A B^{2}}-\frac{B^{\prime} C^{\prime} F}{4 B^{2} C}+\frac{A^{\prime} C^{\prime} F}{4 A B C}-\kappa(p+\rho)=0
$$

$\frac{A^{\prime \prime} F}{2 A B}-\frac{A^{\prime 2} F}{4 A^{2} B}-\frac{B^{\prime \prime} F}{2 B^{2}}+\frac{B^{\prime 2} F}{2 B^{3}}-\frac{A^{\prime} B^{\prime} F}{4 A B^{2}}-\frac{B^{\prime} C^{\prime} F}{4 B^{2} C}+\frac{A^{\prime} C^{\prime} F}{4 A B C}+\frac{A^{\prime} F^{\prime}}{2 A B}-\frac{B^{\prime} F^{\prime}}{2 B^{2}}-\kappa(p+\rho)=0$

These are the three non-linear ordinary differential equations in which six unknown variables $A, B, C, F, \rho$ and $p$ are involved. We can not find the solution of these equations directly. Condition of constant curvature has been used to solve these equations.

\section{Constant curvature solution}

Let's say, for constant curvature $R=R_{0}$, it is apparent that the first and second derivatives of $F(R)=\frac{d f(R)}{d R}$ will always reduce to:

$$
F^{\prime}\left(R_{0}\right)=0=F^{\prime \prime}\left(R_{0}\right)
$$

In the Equation (23), the Equation (20)-(22) and Equation (14) reduce to

$$
\begin{gathered}
\frac{A^{\prime} C^{\prime} F}{4 A B C}-\frac{B^{\prime \prime} F}{2 B^{2}}+\frac{B^{\prime 2} F}{2 B^{3}}-\frac{C^{\prime \prime} F}{2 B C}+\frac{C^{\prime 2} F}{4 B C^{2}}+\frac{A^{\prime} B^{\prime} F}{4 A B^{2}}+\frac{B^{\prime} C^{\prime} F}{4 B^{2} C}-\kappa(p+\rho)=0 \\
\frac{A^{\prime \prime} F}{2 A B}-\frac{A^{\prime 2} F}{4 A^{2} B}-\frac{C^{\prime \prime} F}{2 B C}+\frac{C^{\prime 2} F}{4 B C^{2}}-\kappa(p+\rho)=0
\end{gathered}
$$

$$
\frac{A^{\prime \prime}}{A B}-\frac{A^{\prime 2}}{2 A^{2} B}+\frac{B^{\prime \prime}}{B^{2}}-\frac{B^{\prime 2}}{B^{3}}+\frac{C^{\prime \prime}}{B C}-\frac{C^{\prime 2}}{2 B C^{2}}+\frac{A^{\prime} C^{\prime}}{2 A B C}-R_{0}=0
$$

We will solve these equations using power law as well as exponential law assumptions.

\section{Power Law Assumption} $A \propto r^{m}, B \propto r^{n}$ and $C \propto r^{q}, \quad$ where $m, n$ and $q$ are any real numbers. Therefore, we use $A=k_{1} r^{m}, B=k_{2} r^{n}$, and $C=k_{3} r^{q}$ where $k_{1}, k_{2}$ and $k_{3}$ are constants of proportionality. By inserting these values of $A, B$ and $C$ in Equations (24)-(26) and subtracting them, we attain

$$
\begin{aligned}
& m^{2}-2 m-2 n-m n-n q-m q=0, \\
& m^{2}-2 m+q^{2}-2 m n-2 n q-2 q=0, \\
& q^{2}-2 q+2 n+m q-m n-n q=0 .
\end{aligned}
$$

Also when we put these values in Equations (27) and compare coefficient, we obtain

$$
m^{2}-2 m-2 n+q^{2}-2 q+m q=0 .
$$

To solve these equations, we consider the following cases

I. $m=0, n=q$ II. $n=0, m=q$, III. $q=0, m=n$
Power law assumption is used to solve these equations i.e., 


\section{Case I:}

When we put $m=0, n=q$ in Equation (31), we obtain the following equation

$$
q^{2}-4 q=0
$$

which gives two cases, i.e., either $q=0$ or $q=4$. In former case we get trivial solution while the later case yields the non-trivial solution, given as

$d s^{2}=k_{1} d t^{2}-k_{2} r^{4} d \rho^{2}-k_{3} r^{4} d \phi^{2}-k_{2} r^{4} d z^{2}$.

For this solution it is evaluated that:

$R=0$,

$\rho=\frac{4 F}{\kappa(1+\omega) k_{2} r^{6}}$,

which is a non-vacuum solution.

\section{Case II:}

In this case, we insert $n=0, m=q$ in Equation (31) and have

$3 m^{2}-4 m=0$,

which gives two cases, i.e., either $m=0$ or $m=\frac{4}{3}$. In first case we get trivial solution while in second case we have the following nontrivial solution

$$
d s^{2}=k_{1} r^{\frac{4}{3}} d t^{2}-k_{2} d \rho^{2}-k_{3} r^{\frac{4}{3}} d \phi^{2}-k_{2} d z^{2} .
$$

For this solution, the Ricci scalar and the energy density have been evaluated as

$$
\begin{aligned}
& R=0, \\
& \rho=\frac{8 F}{27 \kappa(1+\omega) k_{2} r^{\frac{6}{3}},}
\end{aligned}
$$

which is obviously a non-vacuum solution.

\section{Case III:}

Here, we put $q=0, m=n$ in Equation (31) and obtain that $n^{2}-4 n=0$, which have again two cases either $q=0$ or $q=4$. In previous case, the solution is trivial. But later case yields the following nontrivial, presented as

$$
d s^{2}=k_{1} r^{4} d t^{2}-k_{2} r^{4} d \rho^{2}-k_{3} d \phi^{2}-k_{2} r^{4} d z^{2} .
$$

For this solution, we evaluated $R$ and $\rho$ as

$$
R=0 \text {, }
$$

$$
\rho=\frac{8 F}{\kappa(1+\omega) k_{2} r^{6}} .
$$

Again it proves that the solution is non-vacuum.

\section{Exponential law assumption}

By using exponential law assumption, i.e., inserting $A(r)=e^{2 \mu(r)}, B(r)=e^{2 v(r)}$ and $C(r)=e^{2 \lambda(r)}$ so that Equations (20)-(22) and (14) be

$\left(\mu^{\prime} \lambda^{\prime}-v^{\prime \prime}-\lambda^{\prime 2}-\lambda^{\prime \prime}+\mu^{\prime} v^{\prime}+v^{\prime} \lambda^{\prime}\right) F e^{-2 v}-F^{\prime \prime} e^{-2 v}+v^{\prime} F^{\prime} e^{-2 v}-\kappa(p+\rho)=0$,

$$
\left(\mu^{\prime 2}+\mu^{\prime \prime}-\lambda^{\prime 2}-\lambda^{\prime \prime}\right) F e^{-2 v}+\left(\mu^{\prime}-\lambda^{\prime}\right) F^{\prime} e^{-2 v}-\kappa(p+\rho)=0,
$$

$\left(\mu^{\prime 2}+\mu^{\prime \prime}+\mu^{\prime} \lambda^{\prime}-v^{\prime \prime}-\mu^{\prime} v^{\prime}-v^{\prime} \lambda^{\prime}\right) F e^{-2 v}+\left(\mu^{\prime}-v^{\prime}\right) F^{\prime} e^{-2 v}-\kappa(p+\rho)=0$,

$$
\left(\mu^{\prime 2}+\mu^{\prime \prime}+v^{\prime \prime}+\lambda^{\prime 2}+\lambda^{\prime \prime}+\mu^{\prime} \lambda^{\prime}\right) F e^{-2 v}-\frac{R_{0}}{2}=0 .
$$

Now, we get four non linear differential equations and six unknown $\mu, v, \lambda, F, p$ and $\rho$. By assumption of constant scalar curvature, we calculate the solution of these equations For constant curvature, these equation are as follows

$$
\begin{aligned}
& \left(\mu^{\prime} \lambda^{\prime}-v^{\prime \prime}-\lambda^{\prime 2}-\lambda^{\prime \prime}+\mu^{\prime} v^{\prime}+v^{\prime} \lambda^{\prime}\right) F e^{-2 v}-\kappa(p+\rho)=0, \\
& \left(\mu^{\prime 2}+\mu^{\prime \prime}-\lambda^{\prime 2}-\lambda^{\prime \prime}\right) F e^{-2}-\kappa(p+\rho)=0,
\end{aligned}
$$

$$
\left(\mu^{\prime 2}+\mu^{\prime \prime}+v^{\prime \prime}+\lambda^{\prime 2}+\lambda^{\prime \prime}+\mu^{\prime} \lambda^{\prime}\right) F e^{-2 v}-\frac{R_{0}}{2}=0\left(\mu^{2}+\mu^{\prime \prime}+\mu^{\prime} \lambda^{\prime}-v^{\prime \prime}-\mu^{\prime} v^{\prime}-v^{\prime} \lambda^{\prime}\right) F e^{-2 v}-\kappa(p+\rho)=0,
$$

$$
\left(\mu^{\prime 2}+\mu^{\prime \prime}+v^{\prime \prime}+\lambda^{\prime 2}+\lambda^{\prime \prime}+\mu^{\prime} \lambda^{\prime}\right) F e^{-2 v}-\frac{R_{0}}{2}=0
$$

We take into account the following three cases, in order to solve these equations

$$
\text { I. } \lambda^{\prime}=0, \text { II. } \mu^{\prime}=0 \text {, III. } v^{\prime}=0 \text {. }
$$

The subtraction of Equations (40), (41) from (39) and similarly, the subtraction of Equations (41) from (40). Also comparing coefficient of Equations (42). we have,

$$
\begin{aligned}
& \mu^{2}+\mu^{\prime \prime}+v^{\prime \prime}-\mu^{\prime} v^{\prime}-v^{\prime} \lambda^{\prime}-\mu^{\prime} \lambda^{\prime}=0, \\
& \left.\mu^{\prime 2}+\mu^{\prime \prime}+\lambda^{\prime 2}+\lambda^{\prime \prime}-2 \mu^{\prime} v^{\prime}-2 v^{\prime} \lambda^{\prime}\right)=0, \\
& \lambda^{\prime 2}+\lambda^{\prime \prime}+\mu^{\prime} \lambda^{\prime}-v^{\prime \prime}-\mu^{\prime} v^{\prime}-v^{\prime} \lambda^{\prime}=0, \\
& \mu^{2}+\mu^{\prime \prime}+v^{\prime \prime}+\lambda^{\prime 2}+\lambda^{\prime \prime}+\mu^{\prime} \lambda^{\prime}=0 .
\end{aligned}
$$

\section{Case I:}

We consider the value of $\lambda^{\prime}=0$, It implies that

$$
\lambda=c_{1} \text {, }
$$

where $c_{1}$ is an integration constant. Using this value in Equation (43)-(46), we get

$$
\mu^{\prime 2}+\mu^{\prime \prime}+v^{\prime \prime}-\mu^{\prime} v^{\prime}=0,
$$


$\mu^{\prime 2}+\mu^{\prime \prime}-2 \mu^{\prime} v^{\prime}=0$

$-v^{\prime \prime}-\mu^{\prime} v^{\prime}=0$,

$\mu^{\prime 2}+\mu^{\prime \prime}+v^{\prime \prime}=0$.

By using the Equation (51) into Equation (48), we obtain

$\mu^{\prime} v^{\prime}=0$

Now, we use Equation (52) into Equation (49) and (50), we get

$\mu^{\prime \prime}+\mu^{2}=0$

$v^{\prime \prime}=0$.

We can easily get the solution for the above equations as

$\mu=\ln \left(a_{1} r+a_{2}\right)$,

$v=b_{1} r+b_{2}$.

Thus, we have found the following non-vacuum solution:

$d s^{2}=\left(a_{1} r+a_{2}\right)^{2} d t^{2}-e^{2 b_{1} r+2 b_{2}} d \rho^{2}-c_{2} d \phi^{2}-e^{2 b_{1} r+2 b_{2}} d z^{2}$.

For this solution, we obtain

$R=0$,

$\rho=\frac{2 a_{1} b_{1} F}{\kappa(1+\omega)\left(a_{1} r+a_{2}\right) e^{2 b_{1} r+2 b_{2}}}$.

\section{Case II:}

We have assumed the value of $\mu^{\prime}=0$, It implies that

$\mu=a_{3}$,

where $a_{3}$ is an integration constant. Using this value in Equations

(43)-(46), we get

$v^{\prime \prime}-v^{\prime} \lambda^{\prime}=0$,

$\lambda^{\prime 2}+\lambda^{\prime \prime}-2 v^{\prime} \lambda^{\prime}=0$

$\lambda^{\prime 2}+\lambda^{\prime \prime}-v^{\prime \prime}-v^{\prime} \lambda^{\prime}=0$,

$\lambda^{\prime 2}+\lambda^{\prime \prime}-v^{\prime \prime}-v^{\prime} \lambda^{\prime}=0$.

From the Equations (59) we have

$v^{\prime \prime}=v^{\prime} \lambda^{\prime}$.

Using Equation (63) in Equations (60)-(62). we obtain

$\lambda^{\prime 2}+\lambda^{\prime \prime}-2 v^{\prime \prime}=0$

$\lambda^{\prime 2}+\lambda^{\prime \prime}+2 v^{\prime \prime}=0$.

These equations can be simplified we get

$\lambda=\ln \left(c_{3} r+c_{4}\right)$,

$v=b_{3} r+b_{4}$.

Thus, we get the following non-vacuum solution

$d s^{2}=a_{4} d t^{2}-e^{2 b_{3} r+2 b_{4}} d \rho^{2}-\left(c_{3} r+c_{4}\right)^{2} d \phi^{2}-e^{2 b_{3} r+2 b_{4}} d z^{2}$.
Corresponding this solution has been evaluated as

$$
\begin{aligned}
& R=0, \\
& \rho=\frac{2 b_{3} c_{3} F}{\kappa(1+\omega)\left(c_{3} r+a_{4}\right) e^{2 b_{3} r+2 b_{4}} .} .
\end{aligned}
$$

\section{Case III:}

When $v^{\prime}=0$ we get on integrating that

$v=b_{5}$,

where $b_{5}$ is an integration constant. Substituting this value of $v$ in Equations (40)-(46), we get

$$
\begin{aligned}
& \mu^{2}+\mu^{\prime \prime}-\mu^{\prime} \lambda^{\prime}=0, \\
& \mu^{2}+\mu^{\prime \prime}+\lambda^{\prime 2}+\lambda^{\prime \prime}=0, \\
& \lambda^{\prime 2}+\lambda^{\prime \prime}+\mu^{\prime} \lambda^{\prime}=0, \\
& \lambda^{\prime 2}+\lambda^{\prime \prime}+\mu^{2}+\mu^{\prime \prime}+\mu^{\prime} \lambda^{\prime}=0 .
\end{aligned}
$$

After making use of Equation (71) in (73), we get

$\mu^{\prime} \lambda^{\prime}=0$.

By using last equation in Equation (70) and Equation (72), we obtain

$$
\begin{aligned}
& \mu^{\prime \prime}+\mu^{2}=0, \\
& \lambda^{\prime 2}+\lambda^{\prime \prime}=0,
\end{aligned}
$$

whose solutions can be easily obtained as

$$
\begin{aligned}
& \mu=\ln \left(a_{5} r+a_{6}\right), \\
& \lambda=\ln \left(c_{5} r+c_{6}\right) .
\end{aligned}
$$

Thus, the corresponding values of $\mathrm{A}, \mathrm{B}$ and $\mathrm{C}$ are

$$
\begin{aligned}
& A=\left(a_{5} r+a_{6}\right)^{2}, \\
& B=b_{6}, \\
& C=\left(c_{5} r+c_{6}\right)^{2} .
\end{aligned}
$$

When we use these values in Equation (14), we evaluate the Ricci scalar as

$$
R=\frac{2 a_{5} c_{5}}{b_{6}\left(a_{5} r+a_{6}\right)\left(c_{5} r+c_{6}\right)},
$$

which is not constant. For the sake of constant Ricci scalar, we must take either $a_{5}=0$ or $c_{5}=0$. In first case, we have

$$
\begin{aligned}
& A=a_{6}^{2}, \\
& B=b_{6}, \\
& C=\left(c_{5} r+c_{6}\right)^{2} .
\end{aligned}
$$

Now for the second case, these turn out to be

$$
A=\left(a_{5} r+a_{6}\right)^{2},
$$




$$
\begin{aligned}
& B=b_{6}, \\
& C=c_{6}^{2} .
\end{aligned}
$$

Finally, the corresponding solutions take the forms:

$$
\begin{aligned}
& d s^{2}=a_{6}^{2} d t^{2}-b_{6} d \rho^{2}-\left(c_{5} r+c_{6}\right)^{2} d \phi^{2}-b_{6} d z^{2}, \\
& d s^{2}=\left(a_{5} r+a_{6}\right)^{2} d t^{2}-b_{6} d \rho^{2}-c_{6}^{2} d \phi^{2}-b_{6} d z^{2} .
\end{aligned}
$$

It is mentioned here that the energy density of these solutions vanish and hence these are the vacuum solutions. Energy Density of the Non-Vacuum Perfect Fluid Static Cylindrically Symmetric Solutions. In this portion, we calculate energy density of the nonvacuum perfect fluid static cylindrically symmetric solutions (32), (33) (34), (57) and (68), which is obtained in the context of $f(R)$ theory of gravity in the last portion. We use generalized LandauLifshitz EMC in the framework of $f(R)$ gravity for this purpose. By substituting the value of $g^{00}$, the Equation (9), it will be

$$
\tau^{00}=f^{\prime}\left(R_{0}\right) \tau_{L L}^{00}+\frac{1}{6 \kappa}\left\{\left(f^{\prime}\left(R_{0}\right) R_{0}-f\left(R_{0}\right)\right)\left(\frac{r A^{\prime}}{A^{2}}+\frac{3}{A}\right)\right\}
$$

Now, by calculating the values of $T^{00}$ and $t_{L L}^{00}$ from Equations (11) and (12) respectively and then using in Equation (10), the final expressions of $\tau_{L L}^{00}$, for the solutions (32), (33), (34), (57) and (68), take the form

$$
\begin{aligned}
\tau_{L L}^{00} & =k_{2}^{2} k_{3} r^{12}\left(\rho-\frac{28}{k_{2} \kappa r^{6}}\right), \\
\tau_{L L}^{00} & =k_{2}^{2} k_{3} r^{\frac{4}{3}}\left(\rho-\frac{4}{9 k_{2} \kappa r^{\frac{6}{3}}}\right),
\end{aligned}
$$

$$
\tau^{00}=f^{\prime}\left(R_{0}\right) e^{4 b_{3} r+4 b_{4}}\left(c_{3} r+c_{4}\right)^{2}\left(\rho-\frac{c_{3}^{2}+\left(2 b_{3}^{2}\left(c_{3} r+c_{4}\right)+4 b_{3} c_{3}\right)\left(c_{3} r+c_{4}\right)}{\kappa e^{2 b_{3} r+2 b_{4}}\left(c_{3} r+c_{4}\right)^{2}}\right)+\frac{1}{2 a_{4} \kappa}\left(f^{\prime}\left(R_{0}\right) R_{0}-f\left(R_{0}\right)\right)
$$

To get the final expression for energy density, we have to consider a suitable $f(R)$ model. It is important to mention here that we must be careful in choosing the $f(R)$ model specially when $R=0$. It is because if the model contains the logarithmic function of Ricci scalar $R$ or a linear superposition of $R^{-n}$, where $\mathrm{n}$ is positive integer, then we can not find this EMC. Hence, we consider the following $f(R)$ model

$$
f(R)=R+\varepsilon R^{2},
$$

where $\varepsilon$ is a positive real number. Consequently, the Equations (32), (33), (34), (57) and (68), results the 00-component of generalized Landau-Lifshitz EMC as

$$
\tau^{00}=k_{2}^{2} k_{3} r^{12}\left(\rho-\frac{28}{k_{2} \kappa r^{6}}\right)
$$

$$
\tau^{00}=e^{4 b_{3} r+4 b_{4}}\left(c_{3} r+c_{4}\right)^{2}\left(\rho-\frac{c_{3}^{2}+\left(2 b_{3}^{2}\left(c_{3} r+c_{4}\right)+4 b_{3} c_{3}\right)\left(c_{3} r+c_{4}\right)}{\kappa e^{2 b_{3} r+2 b_{4}}\left(c_{3} r+c_{4}\right)^{2}}\right) \tau^{00}=k_{2}^{2} k_{3} r^{8}\left(\rho-\frac{8}{k_{2} \kappa r^{6}}\right)(107) .
$$

Furthermore, the stability condition for this $f(R)$ model is also satisfied by all these solutions (as $\mathrm{R}=0$ for every solution) as

$$
\begin{aligned}
& \tau_{L L}^{00}=k_{2}^{2} k_{3} r^{8}\left(\rho-\frac{8}{k_{2} \kappa r^{6}}\right) \\
& \tau_{L L}^{00}=c_{2} e^{4 b_{1} r+4 b_{2}}\left(\rho-\frac{2 b_{1}^{2}}{\kappa e^{2 b_{1} r+2 b_{2}}}\right)
\end{aligned}
$$

and

$\tau_{L L 5}^{00}=e^{4 b_{3} r+4 b_{4}}\left(c_{3} r+c_{4}\right)^{2}\left(\rho-\frac{c_{3}^{2}+\left(2 b_{3}^{2}\left(c_{3} r+c_{4}\right)+4 b_{3} c_{3}\right)\left(c_{3} r+c_{4}\right)}{\kappa e^{2 b_{3} r+2 b_{4}}\left(c_{3} r+c_{4}\right)^{2}}\right)$.

When we use Equations (92)-(96) in Equation (91), the 00components of the generalized Landau-Lifshitz EMC turn out respectively to be

$$
\tau^{00}=S f^{\prime}\left(R_{0}\right) k_{2}^{2} k_{3} r^{12}\left(\rho-\frac{28}{k_{2} \kappa r^{6}}\right)+\frac{1}{2 k_{1} \kappa}\left(f^{\prime}\left(R_{0}\right) R_{0}-f\left(R_{0}\right)\right)
$$

$$
\tau^{00}=f^{\prime}\left(R_{0}\right) k_{2}^{2} k_{3} r^{\frac{4}{3}}\left(\rho-\frac{4}{9 k_{2} \kappa r^{\frac{6}{3}}}\right)+\frac{13}{18 k_{1} r^{\frac{4}{3}} \kappa}\left(f^{\prime}\left(R_{0}\right) R_{0}-f\left(R_{0}\right)\right)
$$

$$
\tau^{00}=f^{\prime}\left(R_{0}\right) k_{2}^{2} k_{3} r^{8}\left(\rho-\frac{8}{k_{2} \kappa r^{6}}\right)+\frac{7}{6 k_{1} r^{4} \kappa}\left(f^{\prime}\left(R_{0}\right) R_{0}-f\left(R_{0}\right)\right),
$$

$$
\tau^{00}=f^{\prime}\left(R_{0}\right) c_{2}^{2} e^{4 b_{1} r+4 b_{2}}\left(\rho-\frac{2 b_{1}^{2}}{\kappa e^{2 b_{1} r+2 b_{2}}}\right)+\frac{1}{6 \kappa}\left(f^{\prime}\left(R_{0}\right) R_{0}-f\left(R_{0}\right)\right) \frac{5 a_{1} r+3 a_{2}}{\left(a_{1} r+a_{2}\right)^{2}}
$$

and

$$
\begin{gathered}
\tau^{00}=k_{2}^{2} k_{3} r^{\frac{4}{3}}\left(\rho-\frac{4}{9 k_{2} \kappa r^{\frac{6}{3}}}\right),(104) \\
\tau^{00}=k_{2}^{2} k_{3} r^{8}\left(\rho-\frac{8}{k_{2} \kappa r^{6}}\right) \\
\tau^{00}=e^{4 b_{3} r+4 b_{4}}\left(c_{3} r+c_{4}\right)^{2}\left(\rho-\frac{c_{3}^{2}+\left(2 b_{3}^{2}\left(c_{3} r+c_{4}\right)+4 b_{3} c_{3}\right)\left(c_{3} r+c_{4}\right)}{\kappa e^{2 b_{3} r+2 b_{4}}\left(c_{3} r+c_{4}\right)^{2}}\right),
\end{gathered}
$$

$\tau^{00}=c_{2}^{2} e^{4 b_{1} r+4 b_{2}}\left(\rho-\frac{2 b_{1}^{2}}{\kappa e^{2 b_{1} r+2 b_{2}}}\right)$

and

$$
\frac{1}{\varepsilon\left(1+2 \varepsilon R_{0}\right)}=\frac{1}{\varepsilon}>0
$$




\section{Summary and conclusion}

The objectives of this work are two folded: Firstly we explore the non-vacuum static cylindrically symmetric solutions in $f(R)$ gravity and the energy distribution of the obtain solution for the perfect fluid case, i.e, $\rho, p \neq 0$. For this purpose, we obtain the field equations of $f(R)$ gravity and solve these equations for static cylindrically symmetric spacetimes considering non-vacuum case while using assumption $R=R_{0}=$ constant . We use power law assumption, to solve these equation and obtain three non vacuum solutions as,

$$
\begin{aligned}
& d s^{2}=k_{1} d t^{2}-k_{2} r^{4} d \rho^{2}-k_{3} r^{4} d \phi^{2}-k_{2} r^{4} d z^{2}, \\
& d s^{2}=k_{1} r^{\frac{4}{3}} d t^{2}-k_{2} d \rho^{2}-k_{3} r^{\frac{4}{3}} d \phi^{2}-k_{2} d z^{2}
\end{aligned}
$$

and

$$
d s^{2}=k_{1} r^{4} d t^{2}-k_{2} r^{4} d \rho^{2}-k_{3} d \phi^{2}-k_{2} r^{4} d z^{2}
$$

We solve these equations again for exponential law assumption and examine three cases $\left(\mu^{\prime}=0, v^{\prime}=0, \lambda^{\prime}=0\right)$ and get three solutions from which one is vacuum while the other two are non vacuum solutions, which are given respectively as,

$$
d s^{2}=\left(a_{1} r+a_{2}\right)^{2} d t^{2}-e^{2 b_{1} r+2 b_{2}} d \rho^{2}-c_{2} d \phi^{2}-e^{2 b_{1} r+2 b_{2}} d z^{2}
$$

and

$$
d s^{2}=a_{4} d t^{2}-e^{2 b_{3} r+2 b_{4}} d \rho^{2}-\left(c_{3} r+c_{4}\right)^{2} d \phi^{2}-e^{2 b_{3} r+2 b_{4}} d z^{2} .
$$

Secondly, we evaluate the energy density of these obtained solutions by using generalized Landau-Lifshtiz EMC of $f(R)$ gravity for a suitable $f(R)$ model and we obtain a well defined expression for components of energy densities of these solution in this case. Moreover, it is also checked that the stability conditions are fulfilled by these solutions.

\section{Acknowledgements}

None.

\section{Conflict of interest}

The author declares no conflict of interest.

\section{References}

1. Weyl H. Eine neue erweiterung der relativitaetstheorie. Annalen der Physik. 1919;364(10):101-133.

2. Eddington AS. The mathematical theory of relativity. USA: Cambridge University Press; 1923.

3. Buchdahl HA. Non-linear Lagrangians and cosmological theory Monthly Notices of the Royal Astronomical Society. 1970;150(1):1-8.

4. Sotiriou TP, Faraoni V. $\mathrm{f}(\mathrm{R})$ theories of gravity. Reviews of Modern Physics. 2010;82(1):451-497.

5. Capozziello $\mathrm{S}$, Stabile A, Troisi A. Spherical symmetry in $\mathrm{f}(\mathrm{R})$-gravity. Classical and Quantum Gravity. 2008;25(8).

6. Capozziello S, Stabile A, Troisi A. Spherically symmetric solutions in $\mathrm{f}(\mathrm{R})$ gravity via the Noether symmetry approach. Classical and Quantum Gravity. 2007;25(8).

7. Kainulainen K, Piilonen J, Reijonen V. Spherically symmetric spacetimes in $\mathrm{f}(\mathrm{R})$ gravity theories. Physical Review D. 007;76(2).
8. Multamaki T, Vilja I. Spherically symmetric solutions of modified field equations in $\mathrm{f}(\mathrm{R})$ theories of gravity. Physical Review D. 2006;74(6).

9. Multamaki T, Vilja I. Static spherically symmetric perfect fluid solutions in $\mathrm{f}(\mathrm{R})$ theories of gravity. Physical Review D. 2007;76(6).

10. Hollenstein L, Lobo FS. Exact solutions of $f(R)$ gravity coupled to nonlinear electrodynamics. Physical Review D. 2008;78(12).

11. Shojai A, Shojai F. Some static spherically symmetric interior solutions of $\mathrm{f}(\mathrm{R})$ gravity. General Relativity and Gravitation. 2012;44(1):211-223.

12. Caramês TRP, de Mello EB. Spherically symmetric vacuum solutions of modified gravity theory in higher dimensions. The European Physical Journal C. 2009;64(1):113-121.

13. Sharif M, Rizwana Kausar H. Dust static spherically sym-metric solution in $\mathrm{f}(\mathrm{R})$ gravity. Journal of the Physical Society of Japan. 2011;80(4).

14. Choudhary K, Jha AK, Mishra LN. Buoyancy and Chemical Reaction Effects on MHD Free Convective Slip Flow of New-tonian and Polar Fluid Through Porous Medium in the presence of Thermal Radiation and Ohmic Heating with Dufour Effect. Facta Universitatis (NIS) Series Mathematicsand Informatics. 2018;33(1):1-29.

15. Deepmala. A Study on Fixed Point Theorems for Nonlinear Contractions and its Applications. India: Pt. Ravishankar Shukla University; 2014.

16. Mishra LN. On existence and behavior of solutions to some nonlinear integral equations with Applications. India: National Institute of Technology; 2017;31(7):2081-2091.

17. Mishra VN. Some Problems on Approximations of Functions in Banach Spaces. India: Indian Institute of Technology; 2007.

18. Mishra VN, Mishra LN. Trigonometric Approximation of Signals (Functions) in Lp (p-1) norm. International Journal of Contemporary Mathematical Sciences. 2012;7(19):909-918.

19. Sharif M, Shamir MF. Plane Symmetric Solutions in $f(R)$ Gravity. Modern Physics Letters A. 2010;25(15):1281-1288.

20. Amir MJ, Maqsood S. Non-Vacuum Plane Symmetric Solu- tions and their Energy Contents in $\mathrm{f}(\mathrm{R})$ gravity. International Journal of Theoretical Physics. 2016;55(2):993-1002.

21. Shamir MF. Plane symmetric vacuum Bianchi type III cos- mology in $\mathrm{f}(\mathrm{R})$ gravity. International Journal of Theoretical Physics. 2011;50(3):637-643.

22. Momeni D, Gholizade H. A note on constant curvature solutions in cylindrically symmetric metric $\mathrm{f}(\mathrm{R})$ gravity. International Journal of Modern Physics D. 2009;18(11):1719-1729.

23. Azadi A, Momeni D, Nouri Zonoz M. Cylindrical solutions in metric f(R) gravity. Physics Letters B. 2008;670(3):210-214.

24. Amir MJ, Sattar S. Locally Rotationally Symmetric Vacuum Solutions in $\mathrm{f}(\mathrm{R})$ Gravity. International Journal of Theoretical Physics. 2014;53(3):773-787.

25. Amir MJ, Naheed S. Spatially Homogeneous Rotating Solution in $f(R)$ Gravity and Its Energy Contents. International Journal of Theoretical Physics. 2013;52(5):1688-1695.

26. Sharif $\mathrm{M}$, Arif $\mathrm{S}$. Static cylindrically symmetric interior solutions in $\mathrm{f}(\mathrm{R})$ gravity. Modern Physics Letters A. 2012;27(25):125-138.

27. Virbhadra KS, Parikh JC. Gravitational energy of a stringy charged black hole. Physics Letters B. 1993;317(3):312-314.

28. Sharif, Amir, MJ. Teleparallel version of the stationary axisymmetric solutions and their energy contents. General Relativity and Gravitation. 2007;39(7): 989-1002.

29. Sharif M, Amir MJ. Teleparallel Killing vectors of the Einstein universe. Modern Physics Letters A. 2008;23(13):963-969. 
30. Sharif M, Amir MJ. Teleparallel versions of Friedmann and LewisPapapetrou spacetimes. General Relativity and Gravitation. 2006;38(12):1735-1745.

31. Sharif M, Amir MJ.Teleparallel energy-momentum distri- bution of spatially homogeneous rotating spacetimes. International Journal of Theoretical Physics. 2008;47(6):1742-1750.

32. Sharif M, Amir MJ. Teleparallel energy-momentum distri- bution of static axially symmetric spacetimes. Modern Physics Letters A. 2008;23(37):3167-3177.
33. Sharif M, Amir MJ. Energy-momentum of the Friedmann models in General Relativity and the teleparallel theory of gravity. Canadian Journal of Physics. 2008;86(11):1297-1302.

34. Sharif M, Amir MJ. Teleparallel version of the Levi-Civita vacuum solutions and their energy contents. Canadian Journal of Physics. 2008;86(9):1091-1096.

35. Sharif M, Shamir MF. Energy distribution in $\mathrm{f}(\mathrm{R})$ gravity. General Relativity and Gravitation. 2010;42(6):1557-1569. 